\title{
Endoplasmic reticulum stress as a correlate of cytotoxicity in human tumor cells exposed to difindolylmethane in vitro
}

\author{
Shishinn Sun,, Jing Han, ${ }^{2}$ Walter M. Ralph Jr., ${ }^{1}$ Alamelu Chandrasekaran, ${ }^{1}$ Kai Liu, ${ }^{1}$ \\ Karen J. Auborn, ${ }^{1,3,4}$ and Timothy H. Carter ${ }^{1-3}$ \\ ${ }^{1}$ North Shore-Long Island Jewish Research Institute, Manhasset, NY 11030, USA \\ 2Department of Biological Sciences, St. John's University, Jamaica, NY 11439, USA \\ ${ }^{3}$ Department of Otolaryngology, Long Island Jewish Medical Center, Long Island Campus of Albert Einstein College of Medicine, New Hyde Park, NY 11040, USA \\ ${ }^{4}$ Department of Microbiology and Immunology, Albert Einstein College of Medicine, Bronx, NY 10461, USA
}

\begin{abstract}
The dietary phytochemical indole-3-carbinol (I3C) protects against cervical cancer in animal model studies and in human clinical trials. I3C and its physiologic condensation product diindolylmethane (DIM) also induce apoptosis of tumor cells in vitro and in vivo, suggesting that these phytochemicals might be useful as therapeutic agents as well as for cancer prevention. Deoxyribonucleic acid microarray studies on transformed keratinocytes and tumor cell lines exposed to pharmacologic concentrations of DIM in vitro are consistent with a cellular response to nutritional deprivation or disruptions in protein homeostasis such as endoplasmic reticulum (ER) stress. In this report we investigate whether specific stress response pathways are activated in tumor cells exposed to DIM and whether the ER stress response might contribute to DIM's cytotoxicity. Induction of the stress response genes GADD153, GADD34 and GADD45A, XBP-1, GRP78, GRP94, and asparagine synthase was documented by Western blot and real-time reverse transcriptase-polymerase chain reaction in C33A cervical cancer cells, and induction of a subset of these was also observed in cancer cell lines from breast (MCF-7) and prostate (DU145). The results are consistent with activation of more than 1 stress response pathway in C33A cells exposed to $75 \mu \mathrm{M}$ DIM. Phosphorylation elF2 $\alpha$ was rapidly and transiently increased, followed by elevated levels of ATF4 protein. Activation of IRE1 $\alpha$ was indicated by a rapid increase in the stress-specific spliced form of XBP-1 messenger ribonucleic acid and a rapid and persistent phosphorylation of JNK1 and JNK2. Transcriptional activation dependent on an ATF6-XBP-1 binding site was detected by transient expression in MCF-7, C33A, and a transformed epithelial cell line (HaCaT); induction of the GADD153 (CHOP) promoter was also confirmed by transient expression. Cleavage of caspase 12 was observed in both DIM-treated and untreated C33A cells but did not correlate with cytotoxicity, whereas caspase 7 was cleaved at later times, coinciding with the onset of apoptosis. The results support the hypothesis that cytotoxic concentrations of DIM can activate cellular stress response pathways in vitro, including the ER stress response. Conversely, DIM was especially cytotoxic to stressed cells. Thapsigargin and tunicamycin, agents that induce ER stress, sensitized cells to the cytotoxic effects of DIM to differing degrees; nutrient limitation had a similar, but even more pronounced, effect. Because DIM toxicity in vitro is enhanced in cells undergoing nutritional deprivation and ER stress, it is possible that stressed cells in vivo, such as those within developing solid tumors, also have increased sensitivity to killing by DIM.
\end{abstract}

\section{INTRODUCTION}

Cell stress is recognized as an important physiologic parameter in the response of tumor cells to various thera-

Received 10 September 2003; Accepted 21 November 2003.

Correspondence to: Timothy H. Carter, Tel: 718 990-1656; Fax: 516562 1022; E-mail: cartert@stjohns.edu. peutic modalities (Tomida and Tsuruo 1999; Giatromanolaki and Harris 2001; Hockel and Vaupel 2001). For example, activation of glucose response proteins (GRPs) in solid tumors (Gazit et al 1999a, 1999b; Reddy et al 2002; Shuda et al 2003), as well as induction of other stress response proteins, may underlie the reported resistance of solid tumors to genotoxic therapies (Little et al 1994; 
Harris 2002; Kassem et al 2002; Reddy et al 2003). On the other hand, certain kinds of cell stress also activate apoptotic pathways (Kanko and Tsukamoto 1994; Perz-Sala and Mollinedo 1995; Zinszner et al 1998; Rao et al 2001, 2003), and it should be possible to capitalize on this effect to enhance therapeutic efficacy.

The dietary phytochemical indole-3-carbinol (I3C), derived from cruciferous vegetables such as broccoli and cabbage, reduces the incidence and inhibits the progression of cervical cancer in animal model studies (Jin et al 1999) and in human clinical trials (Bell et al 2000). Additionally, dietary I3C is an effective treatment for recurrent laryngeal papillomatosis (Coll et al 1997; Auborn et al 1998; Rosen et al 1998), which, like cervical cancer, has a papillomavirus-associated etiology. The mechanism of I3C action in these situations can be explained, at least partly, by its ability to modify the cellular response to estrogen (Meng et al 2000; Auborn et al 2003) and by alterations in estrogen metabolism (Bradlow et al 1991; Auborn et al 1998; Newfield et al 1998). However, I3C and its physiologic condensation product diindolylmethane (DIM) also induce apoptosis in a variety of tumor cell lines in vitro (Cover et al 1998; Ge et al 1999; Chen et al 2001; Chinni et al 2001; Hong et al 2002; Nachhon-Kedmi 2003) and in tissues associated with developing tumors when mice are fed I3C (Chen et al 2001). The latter result suggests that in some circumstances DIM may be selectively toxic for cancer cells, an idea that is consistent with the correlation between sensitivity to DIM killing and tumorigenicity in several variant breast cancer-derived cell lines (Howells et al 2002). These observations raise the possibility of using I3C or DIM as a therapeutic agent for some cancers, perhaps at pharmacologic concentrations rather than the lower concentrations obtained from dietary consumption.

Little is known about the mechanism by which DIM or I3C induces apoptosis, other than that it is not dependent on estrogen signaling (Cover et al 1998; Auborn et al 2003). The few relevant biochemical studies to date report a rapid inhibition of cdk6 in cells exposed to I3C, leading to cell cycle arrest (Cover et al 1998; Firestone and Bjeldanes 2003). Deoxyribonucleic acid (DNA) microarray studies from our laboratory using transformed keratinocytes and tumor cell lines show that many genes are rapidly and persistently upregulated by pharmacologic concentrations of DIM (Carter et al 2002). Prominent among these are genes associated with a cellular response to metabolic stresses such as hypoxia (Price et al 1992; reviewed by Giatromanolaki and Harris 2001) starvation for amino acids or glucose (Watowich and Morimoto 1988) or exposure to toxins like tunicamycin (Kohno et al 1993; Pahl and Baeuerle 1995) and thapsigargin (TG) (Price et al 1992; Haze et al 1999). These conditions and agents engage multiple, interconnected regulatory pathways, leading to enhanced expression of a variety of stress response genes (Fafournoux et al 2000; Lee 2001; Okada et al 2002), many of which are protective but some of which can result in apoptosis after prolonged periods of stress (Jiang et al 1994; Kaneko and Tsukamoto 1994; Perez-Sala and Mollinedo 1995; Rao et al 2001). The endoplasmic reticulum (ER) stress response in mammalian cells, like the similar unfolded protein response (UPR) pathway in yeast (Kaufman 1999; Patil and Walter 2001), is activated by conditions or agents that cause accumulation of unfolded or misfolded proteins and involves activation of the ER membrane-associated dual-function protein kinase-ribonuclease IRE1 (Tirasophon et al 1998), the protein endoplasmic-reticulum-resident kinase (PERK) (Harding et al 2000; reviewed by Rutkowski and Kaufman 2003), and the transcription factor ATF6 (Yoshida et al 1998; Haze et al 1999; Wang et al 2000). These 3 "sensor" proteins each initiate signaling cascades that stimulate transcription of stress response genes. The transcription factor ATF6, normally resident in the ER membrane, is activated in the Golgi of ER-stressed cells by S1P and S2P proteases, leading directly to the transcription of genes such as XBP-1, and CHOP (GADD153) by interaction with a specific nucleotide-binding sequence (Wang et al 2000; Yoshida et al 2000), whereas PERK phosphorylates eIF2 $\alpha$ (Harding et al 1999), enhancing translation of the transcription factor ATF4 (Harding et al 2000) even as it represses translation of many other proteins such as cyclin D1 (Brewer and Diehl 2000). Activation of IRE1 $\alpha$ also results in altered splicing of messenger ribonucleic acid (mRNA) for the transcription factor XBP-1 (Yoshida et al 2001), presumably as a result of translocation of the activated IRE1 $\alpha$ from the ER to the nucleus (Yoshida et al 2001; Lee et al 2002). Additionally, activation of IRE1 $\alpha$ may lead to apoptosis by stimulating phosphorylation of c-jun N-terminal kinase (JNK) and p38 mitogen-activated protein kinase (Urano et al 2000; Zhang et al 2001). The ER stress response can include activation of caspase 12 (Nakagawa et al 2000; Yoneda et al 2001), an ER-resident initiator caspase that ultimately activates caspase 3 (Bitko and Barik 2001).

Several additional stress pathways related to nutrient deprivation have been described in the literature. One of them, activated by amino acid starvation and mediated by the GCN2 kinase, is initially separate from the luminal ER stress response (de Haro et al 1996; Sood et al 2000; Zhang et al 2001). However, both GCN2 and PERK phosphorylate translation factor eIF $2 \alpha$, merging the 2 pathways at this point (Harding et al 2000; Okada et al 2002). Hypoxia engages yet another signaling pathway that can be classed as a nutritional stress response, activating both the specific hypoxia-induced transcription factor HIF1 $\alpha$ (Semenza and Wang 1992) and PERK (Hori et al 2002; Koumenis et al 2002). 
We have studied whether specific stress response pathways are activated during tumor cell response to DIM, by looking for biochemical alterations in key signaling molecules. In the present communication we confirm induction of stress response genes and show that the pattern of signal transduction is consistent with the combined effects of several stress pathways, including the ER stress response (UPR). Furthermore, inducing ER stress by drugs and by nutrient limitation sensitizes cells to killing by DIM, suggesting that this dietary phytochemical may be selectively toxic to cells within developing tumors, which are nutritionally stressed.

\section{MATERIALS AND METHODS}

\section{Reagents}

TG, tunicamycin, and adriamycin were purchased from Sigma (St Louis, MO). 3,3'-Diindolylmethane was a gift from Dr M. Zeligs (BioResponse Inc., Boulder, CO).

\section{Cell lines and cell culture}

C33A, a cervical cancer cell line (human papillomavirus negative, mutant p53), HaCat (a spontaneously immortalized, p53-negative, nontumorigenic human epithelial cell line), and MCF-7 (a human breast cancer cell line with wild-type p53) were obtained from the American Type Culture Collection (Manassas, VA). All cells were maintained as monolayer cultures at $37^{\circ} \mathrm{C}$ and $7 \% \mathrm{CO}_{2}$ in Dulbecco modified Eagle medium (DMEM) (GIBCO-BRL, Gaithersburg, MD) containing $4.5 \mathrm{~g} / \mathrm{L}$ glucose and bicarbonate supplemented with $110 \mathrm{mg} / \mathrm{L}$ sodium pyruvate, $200 \mathrm{mmol} / \mathrm{L}$ glutamine, $100 \mathrm{~g} / \mathrm{L}$ fetal bovine serum, and $100 \mathrm{u} / \mathrm{mL}$ each of penicillin and streptomycin. For combined stress conditions cells were fed with leucine-free DMEM containing 1/50 volume of complete DMEM, 10 $\mathrm{mM}$ glucose, $1.5 \mathrm{mM}$ 2-deoxyglucose, and $100 \mu \mathrm{M} \mathrm{CoCl}_{2}$.

\section{Cell viability}

Cells were trypsinized, seeded at $10^{3}$ cells / well in 96-well plates containing $100 \mu \mathrm{L}$ medium/well, and incubated overnight. The next day, the medium was changed to 100 $\mu \mathrm{L}$ containing either DIM or dimethyl sulfoxide (DMSO) as solvent control. Viability was determined at the indicated times by mitochondrial function (reduction of 3(4,5-dimethylthiazole-2-yl)-2,5-biphenyl tetrazolium bromide), using the CellTiter 96 kit (Promega, Madison, WI) according to the manufacturer's instructions. Absorbance at $595 \mathrm{nM}$ of the solution in individual wells was determined with a multiwell plate reader.

Protein concentration was measured with the MicroBCA kit (Pierce Inc. Rockford, IL), using a bovine serum albumin standard.

\section{Western blotting}

C33A cells were treated with $75 \mu \mathrm{M}$ DIM, 0.075\% DMSO (vehicle control), or antibiotics for various times and lysed on ice in buffer containing $20 \mathrm{mM} \mathrm{N}$-2-hydroxyethylpiperazine- $N^{\prime}$-2-ethane-sulfonic acid, $\mathrm{pH} 7.9,1 \%$ Triton X-100, 0.4 M NaCl, $1 \mathrm{mM}$ dithiothreitol, protease inhibitor cocktail "Complete" (Roche, Mannheim, GR), and phosphatase inhibitors (20 mM $\beta$-glycerophosphate, $1 \mathrm{mM}$ sodium orthovanadate, $30 \mathrm{mM}$ sodium fluoride). Forty micrograms of lysate protein from each sample were analyzed by sodium dodecyl sulfate-polyacrylamide gel electrophoresis. Separated polypeptides were transferred to a nitrocellulose membrane. Before incubation with antibodies, the membrane was blocked with TBST-milk (20 mmol/L Tris- $\mathrm{HCl}, 137 \mathrm{mM} \mathrm{NaCl}, 1.5 \%$ nonfat dry milk, and $0.1 \%$ Tween20, $\mathrm{pH}$ 7.6) for 1 hour. After exposure to antibodies, the membranes were washed in TBST-milk and incubated with horseradish peroxidase (HP)-conjugated anti-mouse IgG antibody (Santa Cruz Biotech, Santa Cruz, CA) at 1:2000 dilution for 1 hour at room temperature. Antibody bound to protein was detected using the enhanced chemiluminescence system (Amersham Life Science, Piscataway, NJ). Membranes were stripped and reprobed with a different primary antibody when necessary. Antibodies against phospho-eIF $2 \alpha$, eIF $2 \alpha$, the cleaved and uncleaved forms of caspase 12 (\#2202) and caspase 7 (\#9492), phospho-JNK, and JNK were from Cell Signaling Technology (Beverly, MA). Anti- $\beta$-actin was from Santa Cruz Biotech.

\section{Transfection and luciferase assays}

Cells grown in monolayer in 60-mm dishes as described above were transfected by using Oligofectin (Invitrogen, Carlsbad, CA) reagent to transport DNA. Each transfection mixture contained $0.5 \mu \mathrm{g}$ of firefly luciferase reporter gene, $0.1 \mu \mathrm{g}$ of pRLGL3 Renilla luciferase expression vector as an internal control, and $9.4 \mu \mathrm{g}$ of total RNA (tRNA) to give a total of $10 \mu \mathrm{g}$ of nucleic acid. Cells were transfected for 16-24 hours and then treated with $100 \mu \mathrm{M}$ DIM, $1 \mu \mathrm{M}$ TG, or $0.1 \%$ DMSO for 24 hours. The transfected cells were lysed and assayed sequentially for firefly and Renilla luciferase activity using a dual luciferase kit (Promega). Results were normalized to the Renilla luciferase activity of the internal control. Each experiment was done in triplicate and the results expressed as the mean. The Renilla luciferase expression vector driven by the HSVTK promoter (pRLTK) was purchased from Promega. Firefly luciferase expression vectors driven by the minimal c-fos promoter were a gift from R. Prywes (Columbia University, New York). P5XATF6GL3 firefly luciferase expression vector including 5 tandem repeats of a stress-activated sequence motif capable of binding ATF6 
and IRE-activated XBP-1 (Yoshida et al 2001), CTCGAGACAGGTGCTGACGTGGCATTC, was cloned upstream of the minimal c-fos promoter at the XhoI site. The same vector with the ATF6-binding sites excised was used as a negative control. The GADD153 promoter-driven luciferase expression vector containing $2.8 \mathrm{~kb}$ upstream of the transcriptional start site was constructed from GADD153CAT (Park et al 1992) by digestion with KpnI and HindIII and insertion into pGL3 basic luciferase vector (Promega).

\section{Real-time polymerase chain reaction}

mRNA expression of stress response genes was measured by quantitative real-time polymerase chain reaction (PCR) using the TaqMan 5' exonuclease assay. One microgram of RNA from each sample was used to prepare complementary DNA (cDNA) in a $50-\mu \mathrm{L}$ reaction, and $5 \mu \mathrm{L}$ of cDNA from each sample was used for real-time PCR. Real-time PCR was performed using the TaqMan PCR core reagent kit and the ABI Prism 7700 sequence detection system (Applied Biosystems, Foster City, CA). The reaction mixture contained $3.5 \mathrm{mM} \mathrm{MgCl}, 0.2 \mathrm{mM}$ each of deoxyadenosine triphosphate, deoxycytidineadenosine triphosphate, and deoxyguanosine triphosphate, $0.4 \mathrm{mM}$ of deoxyuridine triphosphate, $500 \mathrm{nM}$ of forward primer, $500 \mathrm{nM}$ of reverse primer, $100 \mathrm{nM}$ of TaqMan probe, 0.25 $\mathrm{U}$ of uracil DNA glycosylase (AmpErase UNG), and 0.625 $\mathrm{U}$ of Taq polymerase (AmpliTaq Gold) in $1 \times$ TaqMan buffer in a final volume of $25 \mu \mathrm{L}$. Thermal cycler conditions were $50^{\circ} \mathrm{C}$ for 2 minutes, $95^{\circ} \mathrm{C}$ for 10 minutes, and 45 cycles of $95^{\circ} \mathrm{C}$ for 0.3 minutes and $60^{\circ} \mathrm{C}$ for 1 minute. Relative gene expression were determined by delta delta CT method (User Bulletin-2, ABI Prism 7700, Applied Biosystems). $\beta$-Actin was used as an endogenous control. Fold difference in gene expression level was calculated by normalizing to the expression level in DMSO-treated cells. TaqMan primer-probe sequences were GADD34: forward primer CGACTGCAAAG CGGC, reverse primer CAGGAAATG GACAGTGACCTTCT, TaqMan probe TET-CAAGCGCCCAGAAAC CCCTACTCATG-TAMRA; GADD153: forward primer CTGAATCTGCACCAAGCA TGA, reverse primer AAGGTGGGTAGTGTGGCCC, TaqMan probe TET-CAATTG GGAGCATCAGTCCCCCA CT-TAMRA; GADD45 $\alpha$ : forward primer AAGTGCTCAG CAAAGCCCTG, reverse primer GCTTGGCCGCTTCGT ACA, TaqMan probe TET-TCAGCGCACGATCACTGTC GGG-TAMRA; ATF4: forward primer TGGCTGGCT GTGGATGG, reverse primer TCCCGGAGAAGGCATC CT, TaqMan probe TET-TGG TCAGTCCCTCCAACAAC AGCAAG-TAMRA; HPA5 (GRP94): forward primer GTG GAGATCATCGCCAACG, reverse primer AGGAGTGAAGGCGACATAGGAC, TaqMan probe TET-TCAGGGCAACCGCATCACGC-TAMRA; XBP-1: forward primer GCGCCTCACGCACCTG, reverse primer GCTG CTACTCTGTTTTTCAGTTTCC, TaqMan probe TETCCCCGAGGAGAAGGCGCTGAG-TAMRA; HP70 (GRP 78): forward primer TCCTGCGTCGGCGTGT, reverse primer GTTGCCCTGATCGTTGG C, TaqMan probe TETAAGAACGGCCGCGTGGAGATCAT-TAMRA; $\beta$-actin: forward primer CCTGGCACCCAGCACAAT, reverse primer GCCGATCCACA CGGAGTACT, TaqMan probe TET-ATCAAGATCATTGCTCCTCCTGAGCGC-TAMRA.

\section{Detection of XBP-1 mRNA splicing}

Detection of XBP-1 mRNA splicing was done according to published methods (Yoshida et al 2001). tRNA was isolated from DIM-treated C33A cells or from TG-treated HeLa cells using the RNeasy Mini Kit (Qiagen, Valencia, CA). First-strand cDNA was synthesized from $5 \mu \mathrm{g}$ of tRNA by oligo(dT)-primed reverse transcription. PCR containing the forward primer 5'CCTTGTAGTTGAGAACCAGG3' and the reverse primer 5'GGGGCTTGG TA TATATGTGG3' (Yoshida et al 2001) was allowed to continue for 35 cycles. PCR products were separated on a $3 \%$ or $3.5 \%$ agarose gel. Unspliced XBP- 1 mRNA gives rise to a 442-base pair (bp) product, whereas the spliced form produces a 416-bp fragment.

\section{RESULTS}

\section{DIM upregulates stress response genes}

Our previous DNA microarray studies suggested that cellular mRNA content from genes associated with ER stress response was increased within 4-6 hours of exposure to DIM in a group of keratinocyte cell lines, including the cervical cancer line C33A; upregulation of several of these genes (GADD153, ATF3, and C/EBP- $\beta$ ) was documented by Western blot and semiquantitative reverse transcriptase (RT)-PCR (Carter et al 2002). To determine whether other major stress-associated genes are also upregulated by DIM, we used real time to quantify mRNA for GADD153, GADD45A, ATF4, GADD34, GRP78, GRP94, XBP-1, and asparagine synthase. C33A monolayer cultures were treated with either $75 \mu \mathrm{M}$ DIM or DMSO (solvent control) for 6 and 16 hours before extraction of tRNA. Figure 1A shows that RNA from the entire panel of stress response genes was increased at least 2-fold at 6 hours after addition of DIM to the cultures and that expression of most was further elevated at 16 hours. Comparable results were obtained for the growth arrest and DNA-damage associated (GADD) and GRP genes by realtime RT-PCR with MCF-7 breast cancer cells and DU145 prostate cancer cells (Fig 1B). 

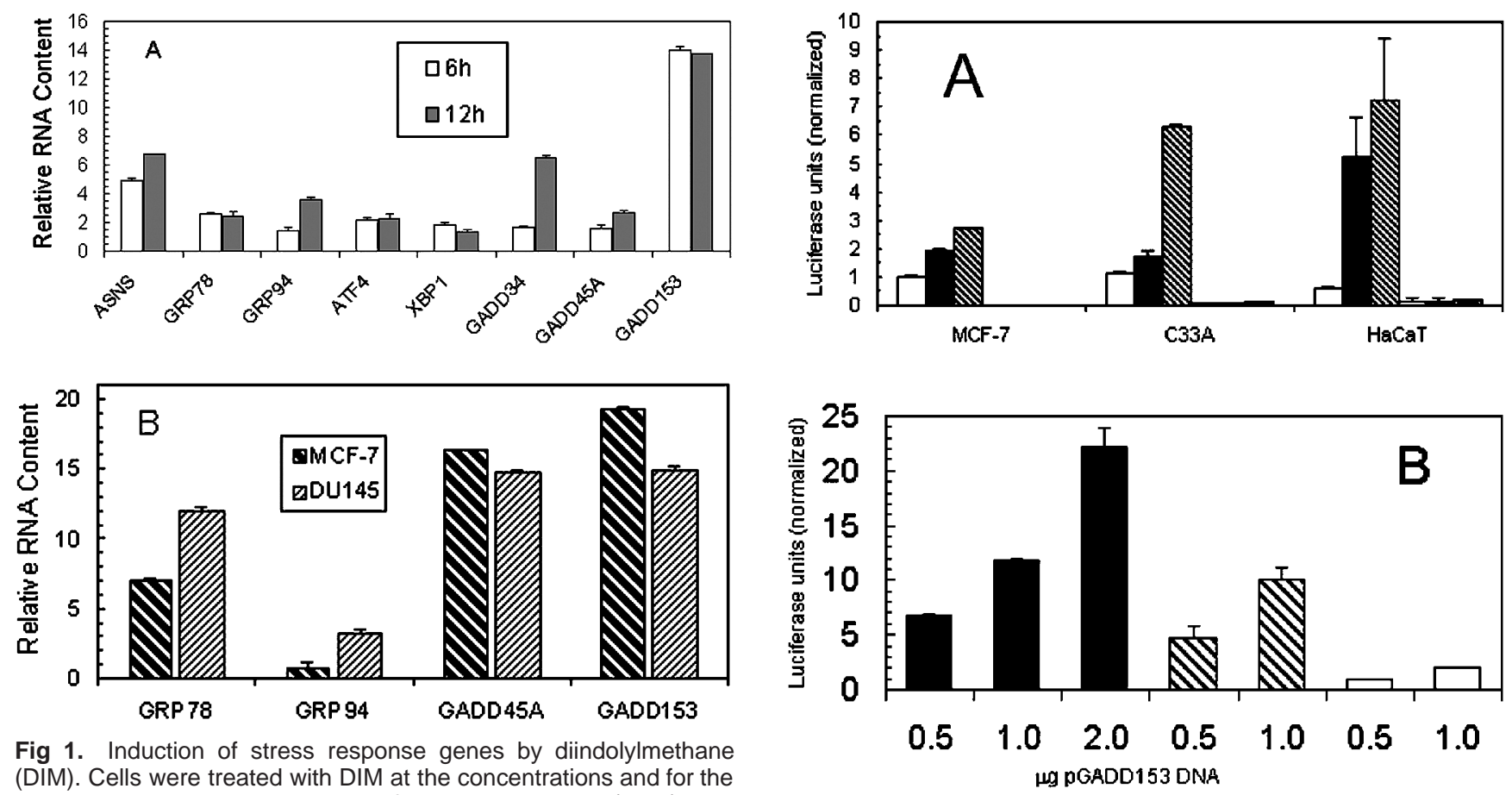
Fig 1. Induction of stress response genes by diindolylmethane
(DIM). Cells were treated with DIM at the concentrations and for the periods indicated, and gene-specific ribonucleic acid (RNA) was quantified by real-time reverse transcriptase-polymerase chain reaction as described in the Materials and Methods. Values were normalized to expression in dimethyl sulfoxide (solvent)-treated cultures, set as 1.0. Panel A: C33A cells were treated with $75 \mu \mathrm{M}$ DIM for 6 hours (white bars) or 16 hours (black bars). RNA from 2 independent sets of duplicate cultures was analyzed and the mean values plotted. Error bars indicate the range of values between the 2 experiments. Panel B: MCF-7 cells (dark bars) and DU145 cells (light bars) were treated with $100 \mu \mathrm{M}$ DIM for 16 hours, and the gene-specific RNAs were quantified as above. Error bars, which are barely visible on the figure, represent the range of duplicate determinations from a single experiment.

\section{DIM treatment leads to functional activation of stress- responsive transcription factors}

Because upregulation of mRNA could result from increased stability as well as a higher rate of transcription, we tested whether DIM could activate a reporter construct consisting of 5 repeats of a synthetic ATF6-XBP-1 binding site upstream of the minimal c-fos promoter (Wang et al 2000), termed a "UPRE" (Yoshida et al 2001) to distinguish it from natural ATF6-binding sites (ERSEs). Transient expression of the reporter plasmid in 2 cancer cell lines (MCF-7 and C33A) as well as in transformed human epithelial cells (HaCaT) revealed that luciferase expression was consistently activated by DIM, although the level of induction was less than that seen with the potent inducer of ER stress, TG (Fig 2A). A luciferase expression plasmid containing an extended natural promoter sequence from the stress response gene GADD153 (CHOP) (Park et al 1992) was also activated in DIM-treated C33A cells (Fig 2B).

Fig 2. Diindolylmethane (DIM) activates transcription from endoplasmic reticulum stress-responsive promoters. Panel A: Cells growing in monolayer were transfected with a firefly luciferase expression plasmid with or without multiple UPRE sites (Wang et al 2000; Yoshida et al 2001), together with a Renilla luciferase expression vector driven by the TK promoter as described in the Materials and Methods, and treated as indicated below. Cell extracts were assayed for both luciferase activities, and the firefly results were normalized to Renilla luciferase expression as described in the Materials and Methods. The first 3 bars of each set are expression from the UPRE-driven plasmid; the second 3 bars are expression from the same plasmid without UPRE sites. Open bars, $0.1 \%$ dimethyl sulfoxide (DMSO); black bars, $100 \mu \mathrm{M}$ DIM; striped bars, $300 \mathrm{nM}$ thapsigargin (TG). Panel B: C33A cells were transfected with GADD153 promoter-driven firefly luciferase and TK-driven Renilla luciferase expression vectors in the amounts indicated and treated with $0.1 \%$ DMSO, $100 \mu \mathrm{M}$ DIM, or $300 \mathrm{nM}$ TG as indicated. After 24 hours, cell extracts were assayed for luciferase and the normalized results calculated as above. Open bars, 0.1\% DMSO; black bars, $100 \mu \mathrm{M}$ DIM; striped bars, 300 nM TG.

\section{DIM activates IRE1 $\alpha$}

One of the early results of ER stress is the activation of transcription factor XBP-1 by alternative splicing catalyzed by the multifunctional protein known as IRE1 $\alpha$ (Yoshida et al 2001; Lee et al 2002). Our DNA microarray data indicated that XBP-1 transcripts were among those elevated between 4 and 12 hours after DIM treatment (Carter et al 2002). We studied whether portions of these transcripts were processed to the ER stress-specific form in C33A cells exposed to DIM. The 2 alternatively spliced forms of XBP-1 mRNA were detected by RT-PCR, using primers that generate different-sized fragments from each splice variant (Yoshida et al 2001). Figure 3A shows that the smaller fragment associated with IRE1 $\alpha$-catalyzed splicing was undetectable in untreated C33A or HeLa 
$\mathrm{Tg}$, DIM,C33A HeLa
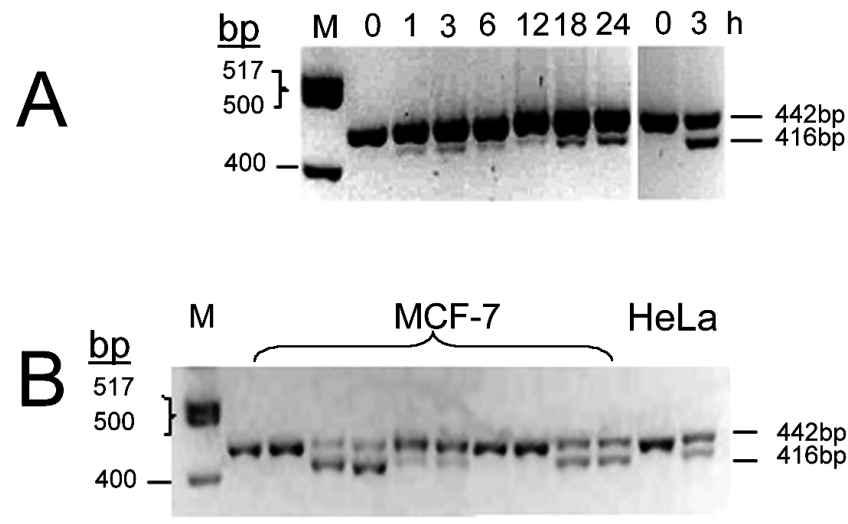

Fig 3. IRE $1 \alpha$-associated alternative splicing of XBP-1 ribonucleic acid (RNA) in diindolylmethane (DIM)-treated cells. Panel A: C33A cells were exposed to $75 \mu \mathrm{M}$ DIM for the indicated times and total RNA analyzed by reverse transcriptase-polymerase chain reaction for alternatively spliced forms as described in the Materials and Methods. HeLa cells treated with $300 \mathrm{nM}$ thapsigargin (TG) were the positive control. bp, base pairs; $\mathrm{M}$, deoxyribonucleic acid-size marker. Panel B: Duplicate MCF-7 and HeLa cultures were treated as indicated for 3 hours and analyzed for XPB-1 RNA.

cells but was present after 1 hour of DIM treatment in C33A. There appeared to be 2 distinct temporal phases of XBP-1 processing in C33A cells after DIM treatment, an early phase peaking at 3 hours and a later, more robust, phase evident at 18 and 24 hours. As expected (Yoshida et al 2001), TG caused a strong response by 3 hours in HeLa cells (Fig 3A) and also in MCF-7 (Fig 3B). Concentrations of TG as low as $5 \mathrm{nM}$, which were only minimally cytotoxic to MCF-7 cells (see Fig 5B), still caused a detectable XBP-1 response in this cell line, as did a 3-hour exposure to DIM (Fig 3B). Adriamycin, a DNAdamaging agent not reported to induce ER stress, did not induce alternative XBP-1 splicing in MCF-7 (Fig 3B).

Activation of IRE1 $\alpha$ has also been reported to result in phosphorylation of JNK (Urano et al 2000; Zhang et al 2001), possibly through interaction with TRAF2 and ASK1 at the ER membrane. Accordingly, we treated C33A cells with DIM and measured JNK phosphorylation at various times during the first 24 hours by using an antibody specific for phosphorylated JNK. Figure 4A shows that the strongest signals for the phosphorylated isoforms JNK1 and JNK2 appeared at 3 hours, the first time point investigated, but persisted for 18 hours. At 24 hours, however, no phosphorylated JNK was detected. The total amounts of immunoreactive JNK remained essentially unchanged during the course of the experiment.

\section{DIM causes elF2 $\alpha$ phosphorylation}

Two nutritional stress pathways converge on the translational regulator $\mathrm{eIF} 2 \alpha$, which can be phosphorylated ei-

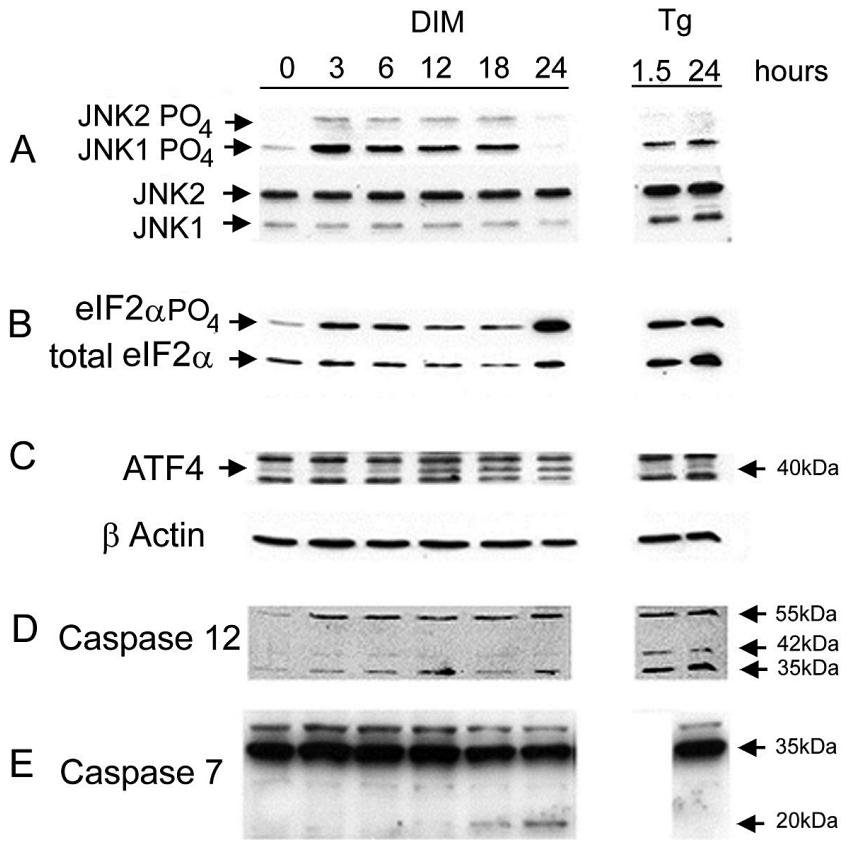

Fig 4. Changes in protein abundance and modification after treatment with diindolylmethane (DIM). C33A cells were treated with 75 $\mu \mathrm{M}$ DIM or $300 \mathrm{nM}$ thapsigargin for the indicated periods and then analyzed by Western blot. Two independent blots were each reprobed for several different proteins and phosphoproteins.

ther by the ER-associated sensor protein kinase PERK or by GCN2 kinase, which is activated by amino acid deprivation. To determine whether $\operatorname{IF} 2 \alpha$ was activated after DIM treatment, C33A cells were exposed to DIM for various time periods in 24 hours, analyzed for phosphorylated immunoreactive eIF2 $\alpha$ by Western blot (Fig 4B), and roughly quantified by densitometry (data not shown). An estimated 15-fold increase in phospho-eIF2 $\alpha$ was apparent at the earliest time point, 3 hours. Like JNK, elevated phosphorylation of eIF2 $\alpha$ persisted for 18 hours, although the total amount of eIF $2 \alpha$ also decreased during this period. Unlike JNK, however, eIF2 $\alpha$ phosphorylation persisted for 24 hours, accompanied by an increase in total eIF2 $\alpha$. TG treatment, done in parallel as a positive control, resulted in phosphorylation of eIF2 $\alpha$, which was detectable at both 90 minutes and 24 hours after exposure to the drug.

\section{DIM upregulates ATF4 protein}

Phosphorylation of eIF2 $\alpha$ has been reported to result in selectively enhanced translation of several proteins, notably the stress-related transcription factor AFT4 or CREB-2 (Harding et al 2000). When Western blots of total protein from cells treated with DIM were analyzed for ATF4, increased signal intensity at the expected mobility was observed after 12 hours (Fig 4C). The same band was 

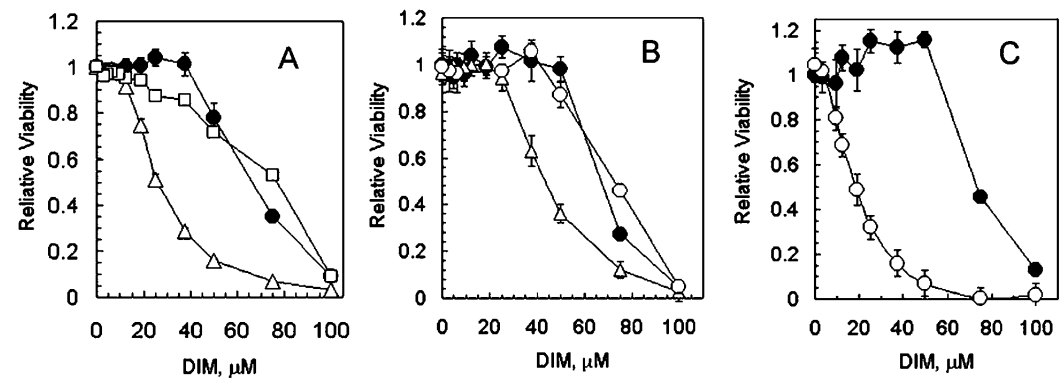

Fig 5. Effect of cell endoplasmic reticulum stress and nutrient limitation on cell viability after treatment with diindolylmethane (DIM). Cells were exposed to varying concentrations of DIM for the indicated periods, and viability was measured as described in the Materials and Methods. Results were normalized to the viability in cultures with drugs or stress conditions but without DIM. Panel A: C33A cells were treated with DIM for 72 hours in the presence of $0.1 \%$ dimethyl sulfoxide (DMSO) (closed circles), $2 \mu \mathrm{g} / \mathrm{mL}$ tunicamycin (open squares), or $300 \mathrm{nM}$ thapsigargin (TG) (open triangles). Cell viability after 72 hours in the absence of DIM was not reduced by either tunicamycin or TG. Panel B: MCF-7 cells were treated with DMSO (closed circles), $100 \mathrm{nM}$ adriamycin (open circles), or $5 \mathrm{nM} / \mathrm{mL}$ TG (open triangles). Cell viability in the absence of DIM was $80 \%$ for adriamycin treatment and $70 \%$ for TG treatment. Panel C: MCF-7 cells were exposed to complete medium (closed circles) or to medium deficient in leucine that also contained 2-deoxyglucose and $\mathrm{CoCl}_{2}$ (open circles), as described in the Materials and Methods. Cell viability in the absence of DIM was $40 \%$ for combined nutrient stress and $70 \%$ for TG treatment.

increased in TG-treated cells, but, as with DIM treatment, the response was not rapid.

\section{Caspase activation in DIM-treated C33A cells}

We have shown previously that caspase 3 is progressively activated in C33A cells after treatment with DIM or I3C starting at around 24 hours. ER stress has been reported to cause apoptosis under certain circumstances, and one of the pathways proposed to mediate this effect is activation of the ER-resident caspase 12 (Nakagawa et al 2000; Rao et al 2001; Morishima et al 2002). Caspase 12 activation has also been proposed to occur as a result of cleavage and activation of caspase 7 (Rao et al 2001). Accordingly, we tested for the amount and cleavage of these 2 caspases by Western blot, as above, using antibodies reported to detect both the uncleaved and the cleaved forms of the enzymes. TG, as expected, caused a rapid apparent net cleavage of caspase 12 into fragments with the mobility expected for activated enzyme (Fig 4D). Interestingly, the amount of uncleaved caspase 12 appeared to increase after a short treatment of cells with either DIM or TG. However, no increase in the fraction of cleaved caspase 12 was evident after DIM treatment. Caspase 7 levels were not greatly changed in cells exposed to either DIM or TG, but cleavage to fragments expected for active enzyme was detected at 18 and 24 hours after DIM treatment but not in cells exposed to TG.

\section{Thapsigargin sensitizes C33A and MCF-7 cells to killing by DIM}

We hypothesized that activation of a luminal ER stress response by DIM is one mechanism by which DIM causes tumor cells in vitro to undergo apoptosis. If this were true, then prestressing the cells, or concomitantly induc- ing ER stress by other means, might be expected to sensitize them to killing by DIM either as an additive effect or as the result of some additional cytotoxic effect of DIM specifically on stressed cells. We tested this prediction by treating C33A and MCF-7 cells with TG, a known inducer of ER stress, at the same time as exposure to increasing concentrations of DIM, and assessing cell viability 72 hours later. Figure 5A shows that for C33A cells, $300 \mathrm{nM}$ TG lowered the $\mathrm{ID}_{50}$ for DIM from $65 \mu \mathrm{M}$ to approximately $25 \mu \mathrm{M}$. TG alone did not reduce cell viability in 72 hours, and pretreatment with TG for 8 or 24 hours also sensitized the cells to killing by DIM in the absence of TG (data not shown). Interestingly, another potent inducer of ER stress, tunicamycin, was not as effective at enhancing DIM cytotoxicity as TG but did enhance C33A cell killing at DIM concentrations below $50 \mu \mathrm{M}$. Minimally toxic concentrations of TG $(5 \mathrm{nM})$ also sensitized the MCF-7 breast cancer cell line to DIM (Fig 5B), whereas $100 \mathrm{nM}$ adriamycin, which alone resulted in a $20 \%$ reduction in cell viability (data not shown), did not alter the sensitivity of MCF-7 cells to DIM.

\section{Combined nutrient limitation and hypoxia signaling sensitizes MCF-7 cells to killing by DIM}

Although some antibiotics are potent inducers of the ER stress response, their effectiveness derives from a highly specific interference with single biochemical processes, such as calcium uptake into the ER in the case of TG. Thus, their modes of action might not be relevant models for the effects of global nutritional limitation, which occurs within solid tumor masses in vivo. Consequently, we studied whether simultaneously limiting 2 essential nutrients, amino acids and glucose, concomitantly with induction of a hypoxic stress response using $\mathrm{CoCl}_{2}$, could sensitize tumor cells to DIM. When MCF-7 cells were 


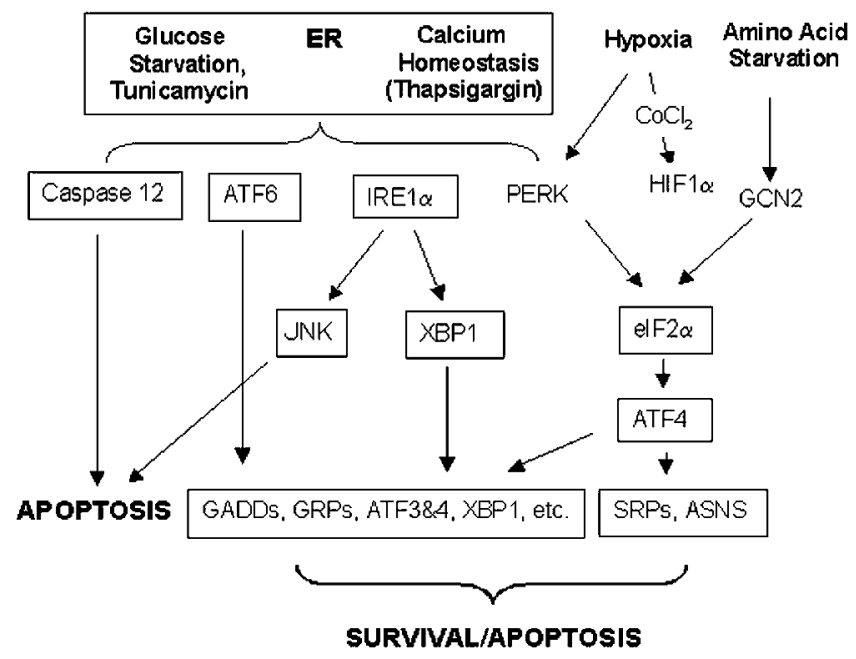

Fig 6. Metabolic stress pathways affected by diindolylmethane (DIM). Abbreviations: ER, endoplasmic reticulum; SRP, multiple specific and general stress response protein. Proteins and genes surrounded by boxes were shown to be affected by DIM.

treated with DIM under these conditions, a dramatic increase in cell killing was observed (Fig 5C), even greater than that obtained with TG. Combined nutrient limitation and $\mathrm{CoCl}_{2}$ themselves reduced cell viability by $60 \%$ after 48 hours, when the experiment was terminated (data not shown). At this time, $75 \mu \mathrm{M}$ DIM was required to kill half the cells in the absence of nutrient limitation, whereas half the remaining viable cells were killed by less than $20 \mu \mathrm{M}$ DIM in the stressed culture.

\section{DISCUSSION}

Previously, we reported upregulation of GRP78, XBP-1, ATF4, ATF3, and GADD153 (CHOP) RNA in a variety of transformed and nontransformed keratinocytes treated for brief periods with DIM. Activation of these genes implied that DIM was causing a response similar to ER stress. Results of the present study strongly support the hypothesis that DIM induces a luminal ER stress response in C33A cells and are consistent with the early activation of IRE1 $\alpha$, although they are also consistent with the activation of other stress response pathways in addition to ER stress. A simplified scheme depicting known pathways of nutritional stress response in mammalian cells is shown in Figure 6, highlighting intermediate steps and endpoints that were affected by DIM in C33A cells. Western blot analysis of cells treated with $75 \mu \mathrm{M}$ DIM demonstrated elevated levels of phosphorylated JNK1 and JNK2 at 3 hours after treatment (Fig 4A), and RT-PCR analysis detected the alternative splicing of XBP-1 mRNA expected from IRE1 $\alpha$ activation (Fig 3A).

At least 3 transcription factors are activated as a proximal result of ER stress: ATF6, XBP-1, and ATF4, each by a discrete posttranscriptional mechanism involving proteolysis, alternative splicing, and translational control, respectively. Considering the experimental evidence for activation of IRE1 $\alpha$ by DIM, the relatively weak transactivation of luciferase expression dependent on tandem repeats of a synthetic IRE-XBP-1 responsive sequence, compared with the activation of this sequence in TGtreated cells, is also consistent with the activation of additional response pathways. The complex biochemical responses to ER stress are regulated by at least 2 other ER transmembrane proteins besides IRE1: ATF6 and PERK (Fig 6). Surviving ER stress is likely to require coordinated, complementary interactions of all 3 factors, causing specific responses that stimulate various groups of ER stress-responsive genes. In this context the cellular response to DIM differs quantitatively, but not qualitatively, from the response to chemical toxins, such as TG, that induce ER stress. Despite its comparatively weak effect in transient expression assays from a UPRE-driven plasmid (Fig 1A), DIM appears to be as good at upregulating GADD153 (CHOP) in cell culture as inducers like tunicamycin or TG (Carter et al 2002). This upregulation is very likely to result from transcriptional induction: when the GADD153 extended promoter region was used to drive luciferase expression in transactivation assay, DIM was at least as efficient an inducer as TG (Fig 1B). The robust response of the "natural" extended promoter compared with the synthetic enhancer element underscores the requirement for multiple signals in the biological context.

Considering the foregoing, it is likely that downstream components of ER stress pathways in addition to XBP-1 are important for the biological effects of DIM on C33A cells in vitro. Phosphorylation of JNK-a protein that is also upregulated by DIM (Carter et al 2002) — can lead to cell death (Xia et al 1995), and elevated JNK phosphorylation was in fact observed after DIM treatment (Fig 4A). A second major pathway for induction of stress response genes is activated by phosphorylation of eIF $2 \alpha$, which enhances translation of the transcription factor ATF4 (CREB-2). This pathway is also activated by DIM in C33A cells. Phosphorylation of eIF2 $\alpha$ on ser 51 , detected by a monoclonal antibody specific for this epitope, occurs within 3 hours of DIM exposure (Fig 4B), and ATF4 polypeptide was visibly elevated on Western blots by 12 hours (Fig 4C). The delayed appearance of ATF4 was contrary to the expectation that it should be a rapid consequence of eIF $2 \alpha$ activation, suggesting that this arm of the ER stress response might not be important for the initial effects of DIM on gene expression, including induction of growth arrest proteins GADD34 and GADD45A, because the genes for these proteins are upregulated by 4-6 hours after exposure to DIM (Fig 1; Carter et al 2002). Alternatively, the available ATF4 antibody, which cross-reacts 
strongly with multiple proteins, may not be sufficiently avid to detect earlier, low-level increases in ATF4.

The pathways responsible for eIF $2 \alpha$ phosphorylation initiated by DIM have not been defined but could occur as a consequence of either PERK phosphorylation (by ER stress or the hypoxia response, for example) or GCN2 activation (by the amino acid starvation pathway), or both. The temporal pattern of several molecular changes documented in the present study is consistent with a biphasic response to DIM, at least in terms of ER stress pathway signaling. Processing of XBP-1 mRNA (Fig 3A) was rapidly but transiently increased, followed by a more pronounced increase after 18-24 hours. On the other hand, phosphorylation of JNK1 and JNK2 was maximal by 3 hours, persisted for 18 hours, and was nearly undetectable at 24 hours. Clearly, a second burst of IRE1 $\alpha$ activity, if indeed that is what is responsible for the late increase in XBP-1 alternative splicing, does not have the same effect on JNK at this time as it did in response to a fresh cellular insult. Similarly, ATF4, a downstream target of eIF $2 \alpha$ phosphorylation, increased detectably only during the second 12-hour period after DIM treatment, at a time when elevated levels of GADD34-induced early by DIM pathways (Carter et al 2002; Fig 1)_should be shutting down ATF4 translation (Novoa et al 2001; Brush et al 2003). Thus, available data are consistent with a complex temporal response of tumor cells to DIM involving more than 1 stress pathway, including those that might already be activated by environmental or genetic factors, or both.

The mechanism by which DIM causes a stress response in tumor cells in vitro is still unknown. The fact that DIM is relatively hydrophobic suggests a potential to interact with cell membranes. However, the high micromolar concentrations required for induction of apoptosis (discussed below) suggest that a high-affinity receptor is not likely to be involved. The only cellular macromolecule for which DIM has so far been identified as a ligand is the aryl hydrocarbon receptor (Jellinck et al 1993). Although this interaction probably underlies many of the effects of DIM on estrogen-dependent cancers (Chen et al 1996; Jin et al 1999; Auborn et al 2003), it can be discounted as the major mechanism for inducing apoptosis in vitro on the basis of genetic experiments (our unpublished data).

Irrespective of how this occurs, the fact that DIM causes an ER stress response, and may activate other stress pathways as well, is consistent with the ability of DIM to induce cells in culture to undergo apoptosis. The mechanism(s) by which this fatal end result is achieved also remains to be determined. Activation of caspase 12 has been proposed to have a role in initiating the apoptotic cascade after ER stress (Nakagawa et al 2000; Rao et al 2002), but this is certainly not the whole story. TG treatment caused a robust cleavage of pro-caspase 12 in C33A cells (Fig 4D) but did not adversely affect viability. Conversely, DIM did not cause a significant increase in the fraction of caspase 12 that had undergone cleavage compared with the untreated cells, yet apoptosis begins at around 24 hours in DIM-treated cultures (Chen et al 2001; Fig 5). Apart from the obvious inhibitory effects stemming from a general cessation of protein synthesis as a result of eIF $2 \alpha$ phosphorylation, perturbation of protein homeostasis in the ER could impair the synthesis or secretion (or both) of key regulatory proteins, growth factors, or cytokines necessary for cell proliferation. Alternatively, the GADD proteins upregulated by DIM, GADD153, GADD45A, and GADD34 are individually capable of directly inhibiting cell growth (Takekawa and Saito 1998; Adler et al 1999; Sarkar et al 2002), and at least one of these, GADD153 (CHOP), has been linked to apoptosis after ER stress (Zinszner et al 1998).

Most of the in vitro cytotoxic effects of DIM, including induction of ER stress, occur at concentrations (eg, 50$100 \mu \mathrm{M})$ that exceed those attained in plasma after dietary consumption of I3C (Stresser et al 1995) but are very similar to the levels of DIM measured in rat liver after oral administration of a single dose of $250 \mathrm{mg} / \mathrm{kg}$ (Anderton et al 2003). This is not surprising considering the fact that physiologic or biochemical activity of agents such as hormones is often only observed in vitro at concentrations that are orders of magnitude higher than those attainable in vivo. Furthermore, accumulation of these agents by cells may decrease the relevance of serum concentration when assessing potential biologic activity. DIM, for example, has been reported to accumulate in the nuclei of breast cancer cells in vitro (Staub et al 2002) as well as in mouse liver and brain in vivo (Anderton et al 2003). However, anticancer effects of DIM in vivo may not require concentrations that induce stress in vitro because tumor cells grown in vitro in the presence of a drug that induces ER stress (eg, TG) become significantly more sensitive to the cytotoxic effects of DIM (Fig 5 A,B), bringing the effective concentrations of DIM more within the range expected for pharmacologic administration. These data are supported by the observation that subjecting MCF-7 cells to the combined stress of limiting amino acids and glucose and to an agent that mimics hypoxia $\left(\mathrm{CoCl}_{2}\right)$ also sensitizes the cells to DIM (Fig 5C).

The stress-responsive GRP promoters are upregulated in developing tumors (Gazit et al 1999a; Reddy et al 2002), and many tumor-derived cell lines exhibit high constitutive levels of stress response proteins such as GRP78 and heat shock proteins (Gazit et al 1999b; Hoang et al 2000; Shuda et al 2003), suggesting that these cells have become adapted to an environment in which they were subjected to nutritional stress. In this context it is noteworthy that untreated C33A cells exhibit basal levels of most of the cell stress endpoints that we examined (eg, 
UPRE-dependent transcription, GRP78 levels, eIF2 $\alpha$ phosphorylation, and JNK1 phosphorylation). It is well established that solid tumors become resistant or refractive to radiotherapy, and some of these stress proteins might exert protective effects with respect to standard chemo- or radiotherapy (Harris 2002; Kassem et al 2002). In light of the increased sensitivity to DIM of several tumor cell lines in which the ER stress pathway has been activated, it is intriguing to speculate that developing cancer cells, or especially cancer cells within solid tumors in vivo (which are expected to be nutritionally stressed), might be especially sensitive to killing by DIM in vivo, either alone or in combination with standard therapeutic modalities. Thus, DIM, or its dietary phytochemical precursor I3C, which has proven effective as a preventive agent for cervical cancer and recurrent respiratory papillomatosis, may also have clinical utility in cancer therapy.

\section{ACKNOWLEDGMENTS}

This work was supported in part by grants CA733850 and CA100967 to K.J.A. and fellowships from the GAANN program at St. John's University (J.H.) and the Robert Wood Johnson Foundation (W.M.R.) grant \#042678.

\section{REFERENCES}

Adler HT, Chinery R, Wu DY, Kussick S, Jr Payne JM, Fornace AJ, Jr Tkachuk DC. 1999. Leukemic HX fusion proteins inhibit GADD34-induced apoptosis and associate with the GADD34 and hNF5/INI1 proteins. Mol Cell Biol 19: 7050-7060.

Anderton MJ, Manson M, Verschoyle RD, Gescher A, Seward W, Williams M. 2003. Pharmacokinetics and tissue disposition of indole-3-carbinol and 33' diindolylmethane after oral administration to mice. Proc Am Assoc Cancer Res 44: 568.

Auborn K, Abramson A, Bradlow HL, Sepkovic D, Mullooly V. 1998. Estrogen metabolism and laryngeal papillomatosis: a pilot study on dietary prevention. Anticancer Res 18: 4569-4573.

Auborn KJ, Fan S, Rosen EM, Goodwin L, Chandraskaren A, Williams D, Chen DZ, Carter T. 2003. Indole-3-carbinol is a negative regulator of estrogen. J Nutr 133(Suppl 7): 2470S-2475S.

Bell MC, Crowley-Nowick P, Bradlow HL, et al. 2000. Placebo-controlled trial of indole-3-carbinol in the treatment of CIN. Gynecol Oncol 78: 123-129.

Bitko V, Barik S. 2001. An endoplasmic reticulum-specific stress-activated caspase (caspase-12) is implicated in the apoptosis of A549 epithelial cells by respiratory syncytial virus. J Cell Biochem 80: 441-454.

Bradlow HL, Michnovicz J, Telang NT, Osborne MP. 1991. Effects of dietary indole-3-carbinol on estrogen metabolism and spontaneous mammary tumors in mice. Carcinogenesis 12: 1571-1574.

Brewer JW, Diehl JA. 2000. PERK mediates cell-cycle exit during the mammalian unfolded protein response. Proc Natl Acad Sci U S A 97: 12625-12630.

Brush MH, Weiser DC, Shenolikar S. 2003. Growth arrest and DNA damage-inducible protein GADD34 targets protein phosphatase 1 alpha to the endoplasmic reticulum and promotes dephos- phorylation of the alpha subunit of eukaryotic translation initiation factor 2. Mol Cell Biol 23: 1292-1303.

Carter TH, Liu K, Ralph W, Jr et al. 2002. Diindolylmethane alters gene expression in human keratinocytes in vitro. J Nutr 132: 3314-3324.

Chen DZ, Qi M, Auborn KJ, Carter TH. 2001. Indole-3-carbinol and diindolylmethane induce apoptosis of human cervical cells and in murine HPV16-transgenic preneoplastic cervical epithelium. J Nutr 131: 3294-3302.

Chen I, Safe S, Bjeldanes L. 1996. Indole-3-carbinol and diindolylmethane as aryl hydrocarbon (Ah) receptor agonists and antagonists in T47D human breast cancer cells. Biochem Pharmacol 51: 1069-1076.

Chinni SR, Li Y, Upadhyay S, Koppolu PK, Sarkar FH. 2001. Indole3-carbinol (I3C) induced cell growth inhibition G1 cell cycle arrest and apoptosis in prostate cancer cells. Oncogene 20: 29272936.

Coll DA, Rosen CA, Auborn K, Potsic WP, Bradlow HL. 1997. Treatment of recurrent respiratory papillomatosis with indole-3-carbinol. Am J Otolaryngol 18: 283-285.

Cover CM, Hieh SJ, Tran SH, Hallden G, Kim GS, Bjeldanes LF, Firestone GL. 1998. Indole-3-carbinol inhibits the expression of cyclin-dependent kinase- 6 and induces a G1 cell cycle arrest of human breast cancer cells independent of estrogen receptor signaling. J Biol Chem 273: 3838-4387.

de Haro C, Mendez R, Santoyo J. 1996. The eIF-2alpha kinases and the control of protein synthesis. FASEB J 10: 1378-1387.

Fafournoux P, Bruhat A, Jousse C. 2000. Amino acid regulation of gene expression. Biochem J 351: 1-12.

Firestone GL, Bjeldanes LF. 2003. Indole-3-carbinol and 3-3'-diindolylmethane antiproliferative signaling pathways control cellcycle gene transcription in human breast cancer cells by regulating promoter-Sp1 transcription factor interactions. J Nutr 133: 2448S-2455S.

Gazit G, Hung G, Chen X, Anderson WF, Lee AS. 1999a. Use of the glucose starvation-inducible glucose-regulated protein 78 promoter in suicide gene therapy of murine fibrosarcoma. Cancer Res 59: 3100-3106.

Gazit G, Lu J, Lee AS. 1999b. De-regulation of GRP stress protein expression in human breast cancer cell lines. Breast Cancer Res Treat 54: 135-146.

Ge X, Fares FA, Yannai S. 1999. Induction of apoptosis in MCF-7 cells by indole-3-carbinol is independent of p53 and bax. Anticancer Res 19: 3199-3203.

Giatromanolaki A, Harris AL. 2001. Tumour hypoxia signaling pathways and hypoxia inducible factor expression in human cancer. Anticancer Res 21: 4317-4324.

Harding HP, Novoa I, Zhang Y, Zeng H, Wek R, Schapira M, Ron D. 2000. Regulated translation initiation controls stress-induced gene expression in mammalian cells. Mol Cell 6: 1099-1108.

Harding HP, Zhang Y, Ron D. 1999. Protein translation and folding are coupled by an endoplasmic-reticulum-resident kinase. $\mathrm{Na}$ ture 397: 271-274.

Harris AL. 2002. Hypoxia-a key regulatory factor in tumour growth. Natl Rev Cancer 2: 38-47.

Haze K, Yoshida H, Yanagi H, Yura T, Mori K. 1999. Mammalian transcription factor ATF6 is synthesized as a transmembrane protein and activated by proteolysis in response to endoplasmic reticulum stress. Mol Biol Cell 10: 3787-3799.

Hoang AT, Huang J, Rudra-Ganguly N, Zheng J, Powell WC, Rabindran SK, Wu C, Roy-Burman P. 2000. A novel association between the human heat shock transcription factor 1 (HF1) and prostate adenocarcinoma. Am J Pathol 156: 857-864. 
Hockel M, Vaupel P. 2001. Biological consequences of tumor hypoxia. Semin Oncol 28(Suppl 8): 36-41.

Hong C, Firestone GL, Bjeldanes LF. 2002. Bcl-2 family-mediated apoptotic effects of 33'-diindolylmethane (DIM) in human breast cancer cells. Biochem Pharmacol 63: 1085-1097.

Hori O, Ichinoda F, Tamatani T, et al. 2002. Transmission of cell stress from endoplasmic reticulum to mitochondria: enhanced expression of Lon protease. J Cell Biol 157: 1151-1160.

Howells LM, Gallacher-Horley B, Houghton CE, Manson MM, Hudson EA. 2002. Indole-3-carbinol inhibits protein kinase B/Akt and induces apoptosis in the human breast tumor cell line MDA MB468 but not in the nontumorigenic HBL100 line. Mol Cancer Ther 1: 1161-1172.

Jellinck PH, Forkert PG, Riddick DS, Okey AB, Michnovicz JJ, Bradlow HL. 1993. Ah receptor binding properties of indole carbinols and induction of hepatic estradiol hydroxylation. Biochem Pharmacol 45: 1129-1136.

Jiang S, Chow SC, Nicotera P, Orrenius S. 1994. Intracellular Ca2+ signals activate apoptosis in thymocytes: studies using the $\mathrm{Ca}(2+)$-ATPase inhibitor thapsigargin. Exp Cell Res 212: 84-92.

Jin L, Qi M, Chen DZ, Anderson A, Yang GY, Arbeit JM, Auborn KJ. 1999. Indole-3-carbinol prevents cervical cancer in human papilloma virus type16 (HPV16) transgenic mice. Cancer Res 59: 3991-3997.

Kaneko Y, Tsukamoto A. 1994. Thapsigargin-induced persistent intracellular calcium pool depletion and apoptosis in human hepatoma cells. Cancer Lett 79: 147-155.

Kassem HS, Sangar V, Cowan R, Clarke N, Margison GP. 2002. A potential role of heat shock proteins and nicotinamide N-methyl transferase in predicting response to radiation in bladder cancer. Int J Cancer 101: 454-460.

Kaufman RJ. 1999. Stress signaling from the lumen of the endoplasmic reticulum: coordination of gene transcriptional and translational controls. Genes Dev 13: 1211-1233.

Kohno K, Normington K, Sambrook J, Gething MJ, Mori K. 1993. The promoter region of the yeast KAR2 (BiP) gene contains a regulatory domain that responds to the presence of unfolded proteins in the endoplasmic reticulum. Mol Cell Biol 13: 877890.

Koumenis C, Naczki C, Koritzinsky M, Rastani S, Diehl A, Sonenberg N, Koromilas A, Wouters BG. 2002. Regulation of protein synthesis by hypoxia via activation of the endoplasmic reticulum kinase PERK and phosphorylation of the translation initiation factor eIF2alpha. Mol Cell Biol 22: 7405-7416.

Lee AS. 2001. The glucose-regulated proteins: stress induction and clinical applications. Trends Biochem Sci 26: 504-510.

Lee K, Tirasophon W, Shen X, et al. 2002. IRE1-mediated unconventional mRNA splicing and S2P-mediated ATF6 cleavage merge to regulate $\mathrm{XBP}-1$ in signaling the unfolded protein response. Genes Dev 16: 452-466.

Little E, Ramakrishnan M, Roy B, Gazit G, Lee AS. 1994. The glucose-regulated proteins (GRP78 and GRP94): functions gene regulation and applications. Crit Rev Eukaryot Gene Expr 4: 118.

Meng Q, Yuan F, Goldberg ID, Rosen EM, Auborn K, Fan S. 2000. Indole-3-carbinol is a negative regulator of estrogen receptoralpha signaling in human tumor cells. J Nutr 130: 2927-2931.

Morishima N, Nakanishi K, Takenouchi H, Shibata T, Yasuhiko Y. 2002. An endoplasmic reticulum stress-specific caspase cascade in apoptosis: cytochome c-independent activation of caspase-9 by caspase-12. J Biol Chem 277: 34287-34294.

Nachhon-Kedmi M, Yannai S, Haj A, Fares FA. 2003. Indole-3-carbinol and 33'-diindolylmethane induce apoptosis in human prostate cancer cells. Food Chem Toxicol 41: 745-752.
Nakagawa T, Zhu H, Morishima N, Li E, Xu J, Yankner BA, Yuan J. 2000. Caspase-12 mediates endoplasmic-reticulum-specific apoptosis and cytotoxicity by amyloid-beta. Nature 403: 98-103.

Newfield L, Bradlow HL, Sepkovic DW, Auborn K. 1998. Estrogen metabolism and the malignant potential of human papillomavirus immortalized keratinocytes. Exp Biol Med 217: 322-326.

Novoa I, Zeng H, Harding HP, Ron D. 2001. Feedback inhibition of the unfolded protein response by GADD34-mediated dephosphorylation of eIF2alpha. J Cell Biol 153: 1011-1022.

Okada T, Yoshida H, Akazawa R, Negishi M, Mori K. 2002. Distinct roles of activating transcription factor 6 (ATF6) and doublestranded RNA-activated protein kinase-like endoplasmic reticulum kinase (PERK) in transcription during the mammalian unfolded protein response. Biochem J 366: 585-594.

Pahl HL, Baeuerle PA. 1995. A novel signal transduction pathway from the endoplasmic reticulum to the nucleus is mediated by transcription factor NF-kappa B. EMBO J 14: 2580-2588.

Park JS, Luethy JD, Wang MG, Fargnoli J, Fornace AJ, Jr McBride OW, Holbrook NJ. 1992. Isolation, characterization and chromosomal localization of the human GADD153 gene. Gene 116: 259-267.

Patil C, Walter P. 2001. Intracellular signaling from the endoplasmic reticulum to the nucleus: the unfolded protein response in yeast and mammals. Curr Opin Cell Biol 13: 349-355.

Perez-Sala D, Mollinedo F. 1995. Inhibition of N-linked glycosylation induces early apoptosis in human promyelocytic HL-60 cells. J Cell Physiol 163: 523-531.

Price BD, Calderwood SK. 1992. Gadd45 and Gadd153 messenger RNA levels are increased during hypoxia and after exposure of cells to agents which elevate the levels of the glucose-regulated proteins. Cancer Res 52: 3814-3817.

Price BD, Mannheim-Rodman LA, Calderwood SK. 1992. Brefeldin A, thapsigargin and AIF4- stimulate the accumulation of GRP78 mRNA in a cycloheximide dependent manner, whilst induction by hypoxia is independent of protein synthesis. J Cell Physiol 152: 545-552.

Rao RV, Hermel E, Castro-Obregon S, del Rio G, Ellerby LM, Ellerby HM, Bredesen DE. 2001. Coupling endoplasmic reticulum stress to the cell death program. Mechanism of caspase activation. J Biol Chem 276: 33869-33874.

Reddy RK, Dubeau L, Kleiner H, et al. 2002. Cancer-inducible transgene expression by the Grp94 promoter: spontaneous activation in tumors of various origins and cancer-associated macrophages. Cancer Res 62: 7207-7212.

Reddy RK, Mao C, Baumeister P, Austin RC, Kaufman RJ, Lee AS. 2003. Endoplasmic reticulum chaperone protein GRP78 protects cells from apoptosis induced by topoisomerase inhibitors: role of ATP binding site in suppression of caspase-7 activation. J Biol Chem 278: 20915-20924.

Rosen CA, Woodson GE, Thompson JW, Hengesteg AP, Bradlow HL. 1998. Preliminary results of the use of indole-3-carbinol for recurrent respiratory papillomatosis. Otolaryngol_-Head Neck Surg 118: 810-815.

Rutkowski DT, Kaufman RJ. 2003. All roads lead to ATF4. Dev Cell 4: 442-444.

Sarkar D, Su ZZ, Lebedeva IV, Sauane M, Gopalkrishnan RV, Valerie K, Dent P, Fisher PB. 2002. Mda-7 (IL-24) mediates selective apoptosis in human melanoma cells by inducing the coordinated overexpression of the GADD family of genes by means of p38 MAPK. Proc Natl Acad Sci U S A 99: 10054-10059.

Semenza GL, Wang GL. 1992. A nuclear factor induced by hypoxia via de novo protein synthesis binds to the human erythropoietin gene enhancer at a site required for transcriptional activation. Mol Cell Biol 12: 5447-5454. 
Shuda M, Kondoh N, Imazeki N, et al. 2003. Activation of the ATF6 XBP-1 and grp78 genes in human hepatocellular carcinoma: a possible involvement of the ER stress pathway in hepatocarcinogenesis. J Hepatol 38: 605-614.

Sood R, Porter AC, Olsen DA, Cavener DR, Wek RC. 2000. A mammalian homologue of GCN2 protein kinase important for translational control by phosphorylation of eukaryotic initiation factor-2alpha. Genetics 154: 787-801.

Staub RE, Feng C, Onisko B, Bailey GS, Firestone GL, Bjeldanes LF. 2002. Fate of indole-3-carbinol in cultured human breast tumor cells. Chem Res Toxicol 15: 101-109.

Stresser DM, Williams DE, Griffin DA, Bailey GS. 1995. Mechanisms of tumor modulation by indole-3-carbinol. Disposition and excretion in male Fischer 344 rats. Drug Metab Dispos 23: 965-975.

Takekawa M, Saito H. 1998. A family of stress-inducible GADD45like proteins mediate activation of the stress-responsive MTK1 / MEKK4 MAPKKK. Cell 95: 521-530.

Tirasophon W, Welihinda AA, Kaufman RJ. 1998. A stress response pathway from the endoplasmic reticulum to the nucleus requires a novel bifunctional protein kinase/endoribonuclease (Ire1p) in mammalian cells. Genes Dev 12: 1812-1824.

Tomida A, Tsuruo T. 1999. Drug resistance mediated by cellular stress response to the microenvironment of solid tumors. Anticancer Drug Des 14: 169-177.

Urano F, Wang X, Bertolotti A, Zhang Y, Chung P, Harding HP, Ron D. 2000. Coupling of stress in the ER to activation of JNK protein kinases by transmembrane protein kinase IRE1. Science 287: 664-666.

Wang Y, Shen J, Arenzana N, Tirasophon W, Kaufman RJ, Prywes R. 2000. Activation of ATF6 and an ATF6 DNA binding site by the endoplasmic reticulum stress response. J Biol Chem 275: 2701327020.

Watowich SS, Morimoto RI. 1988. Complex regulation of heat shockand glucose-responsive genes in human cells. Mol Cell Biol 8: 393-405.
Xia Z, Dickens M, Raingeaud J, Davis RJ, Greenberg ME. 1995. Opposing effects of ERK and JNK-p38 MAP kinases on apoptosis Science 270: 1326-1231.

Yoneda T, Imaizumi K, Oono K, Yui D, Gomi F, Katayama T, Tohyama M. 2001. Activation of caspase-12, an endoplastic reticulum (ER) resident caspase, through tumor necrosis factor receptor-associated factor 2-dependent mechanism in response to the ER stress. J Biol Chem 276: 13935-13940.

Yoshida H, Haze K, Yanagi H, Yura T, Mori K. 1998. Identification of the cis-acting endoplasmic reticulum stress response element responsible for transcriptional induction of mammalian glucose-regulated proteins involvement of basic leucine zipper transcription factors. J Biol Chem 273: 33741-33749.

Yoshida H, Matsui T, Yamamoto A, Okada T, Mori K. 2001. XBP-1 mRNA is induced by ATF6 and spliced by IRE1 in response to ER stress to produce a highly active transcription factor. Cell 107: 881-891.

Yoshida H, Okada T, Haze K, Yanagi H, Yura T, Negishi M, Mori K. 2000. ATF6 activated by proteolysis binds in the presence of NFY (CBF) directly to the cis-acting element responsible for the mammalian unfolded protein response. Mol Cell Biol 20: 67556767.

Zhan Q, Lord KA, Alamo I, Jr et al. 1994. The gadd and MyD genes define a novel set of mammalian genes encoding acidic proteins that synergistically suppress cell growth. Mol Cell Biol 14: 23612371.

Zhang C, Kawauchi J, Adachi MT, Hashimoto Y, Oshiro S, Aso T, Kitajima S. 2001. Activation of JNK and transcriptional repressor ATF3/LRF1 though the IRE1/TRAF2 pathway is implicated in human vascular endothelial cell death by homocysteine. Biochem Biophys Res Commun 289: 718-724.

Zinszner H, Kuroda M, Wang X, Batchvarova N, Lightfoot RT, Remotti H, Stevens JL, Ron D. 1998. CHOP is implicated in programmed cell death in response to impaired function of the endoplasmic reticulum. Genes Dev 12: 982-995. 